materials ${ }^{2}$. However, spin-orbit coupling too is found to be a significant source of spin relaxation ${ }^{3}$.

There are more than 30 million organic compounds differing in structure and composition, resulting in a multitude of features found in organic materials. Even when the same material is used in different experiments, its purity can vary, and any impurities can substantially affect the data. We note that two independent groups used deuterated materials to explore the hyperfine field effects on the electron spin relaxation, and reached opposite conclusions ${ }^{2,4}$.

Furthermore, divergent conclusions from similar experiments ${ }^{5}$ could also be the consequence of dissimilar processes for sample preparations and of the lack of consistency in the testing instruments of different groups, notably in the organic magnetoresistance subfield.

Therefore, priority should be given to standardizing data. Researchers should carefully record experimental procedure, material and so on, and possibly share them in a database repository, so that results from different laboratories can be compared. Such a repository would also be useful in testing applicability and accuracy of theoretical models.
References

1. Boehme, C. \& Lupton, J. M. Nature Nanotech. 8, 612-615 (2013).

2. Nguyen, T. D. et al. Nature Mater. 9, 345-352 (2010)

3. Nuccio, L. et al. Phys. Rev. Lett. 110, 216602 (2013).

4. Rolfe, N. J. et al. Phys. Rev. B 80, 241201 (2009).

5. Bagnich, S. A. et al. J. Appl. Phys. 105, 123706 (2009).

Qiming Peng ${ }^{1 \star}$ and Tianyou Zhang ${ }^{2}$

'State Key Laboratory of Supramolecular

Structure and Materials, Jilin University Qianjin Avenue, Changchun 130012, China, ${ }^{2}$ State Key Laboratory of Luminescence and Applications, Changchun Institute of Optics, Fine Mechanics, and Physics, Chinese Academy of Sciences, Changchun 130033, China.

*e-mail: pengqm12@mails.jlu.edu.cn

\title{
Focusing on the molecular scale
}

To the Editor - The call of Boehme and Lupton ${ }^{1}$ for the increased use of spectroscopy to study spin phenomena in organic spintronics is valuable to the community. However, there are instances where spectroscopy techniques such as $\mathrm{X}$-ray absorption, X-ray magnetic circular dichroism, X-ray photoemission, twophoton photoemission and muon spinrotation spectroscopy are not suitable investigative tools because they provide only an averaged-out response of interface and bulk phenomena. One example is the study of ferromagnet/organic semiconductor interfaces, for which the above methods are not sufficiently sensitive for locally probing the magnetic field-dependent response of the interface. I would argue that the organic spintronics community should tackle the challenges in the field starting from the molecular scale working towards building a theoretical framework of the fundamental physical mechanisms.

For example, the emergent subfield of interface molecular spintronics has a strong theoretical hold supported by advanced spectroscopy tools to probe molecular spin responses. Although the observation of spin-conserved tunnelling through organic semiconductors is accepted in theory, questions remain about the sign and magnitude of tunnelling magnetoresistance in organic tunnel junctions. Spin-polarized scanning tunnelling spectroscopy offers direct access to the spin states of the organic molecules on magnetic surfaces ${ }^{2}$. Supported by computational $a b$ initio studies, such exploration provides direct insight on the role of local surface chemistry in the magnetic response of an interface. Evidence of inversion and amplification of interface spin-polarization may explain the reported discrepancy in tunnelling magnetoresistance values of macroscopic organic tunnelling devices. Recently, reports of induced molecular-magnetism and the spin-filter response at the interface in macroscopic organic devices ${ }^{3}$ have been confirmed using such techniques ${ }^{4}$. They offer a way forward in our understanding and may be technologically relevant for the development of molecular spintronic devices in sensor, memory and computing applications ${ }^{3}$. Also, similar approaches for probing the organic magnetoresistance effect at lower dimensions may help to appropriately scrutinize its origin.

In addition to the message of Boehme and Lupton, other important criticisms ${ }^{5}$ of the field are the occasional improper device fabrication practices for soft and porous organic semiconductors, misinterpretation of experimental reports and the poor reproducibility of the experimental data from different research groups, which raise concerns about the technological applicability of organic spintronic devices. Channelling our research efforts towards the molecular scale may present a promising future for the field.

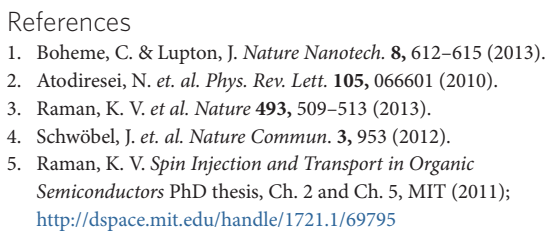

Karthik V. Raman

Solid State and Structural Chemistry Unit, Indian Institute of Science, Bangalore-560012, India. e-mail:kvraman@sscu.iisc.ernet.in

\section{An agnostic approach}

To the Editor - The current situation of research in organic spintronics as outlined by Boehme and Lupton ${ }^{1}$ may be due in part to the early successes ${ }^{2}$ and subsequent heavy reliance on the theory and techniques inherited from the more mature field of inorganic semiconductors. However, there are differences between organic and inorganic semiconductors that reflect in the microscopic reality of spin-dependent processes. Models that account for these differences are therefore necessary to advance our understanding of spin-based phenomena in organic semiconductors.

Discriminating between different models of spin transport and dynamics in organic semiconductors has proven challenging. Recent progress, however, is promising. Harmon and Flatté ${ }^{3}$ have predicted signatures for the three dominant spin-relaxation mechanisms for organic semiconductors: intrasite relaxation, hyperfine interactions and spin-orbit coupling. Similarly, Janssen et al. ${ }^{4}$ have developed a unified model of organic magnetoresistance, which includes polaron-pair recombination, bipolaron spin blocking and triplet exciton-polaron quenching mechanisms. These amalgamated approaches force the characteristics of 